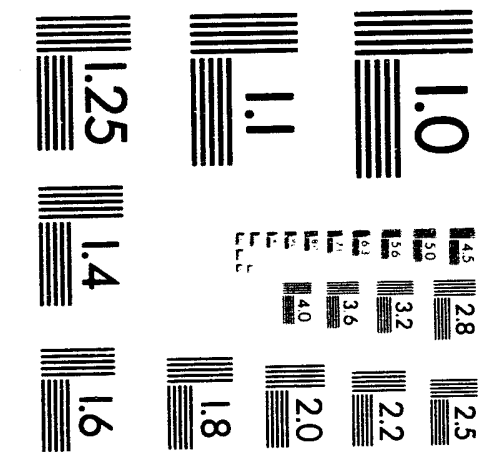



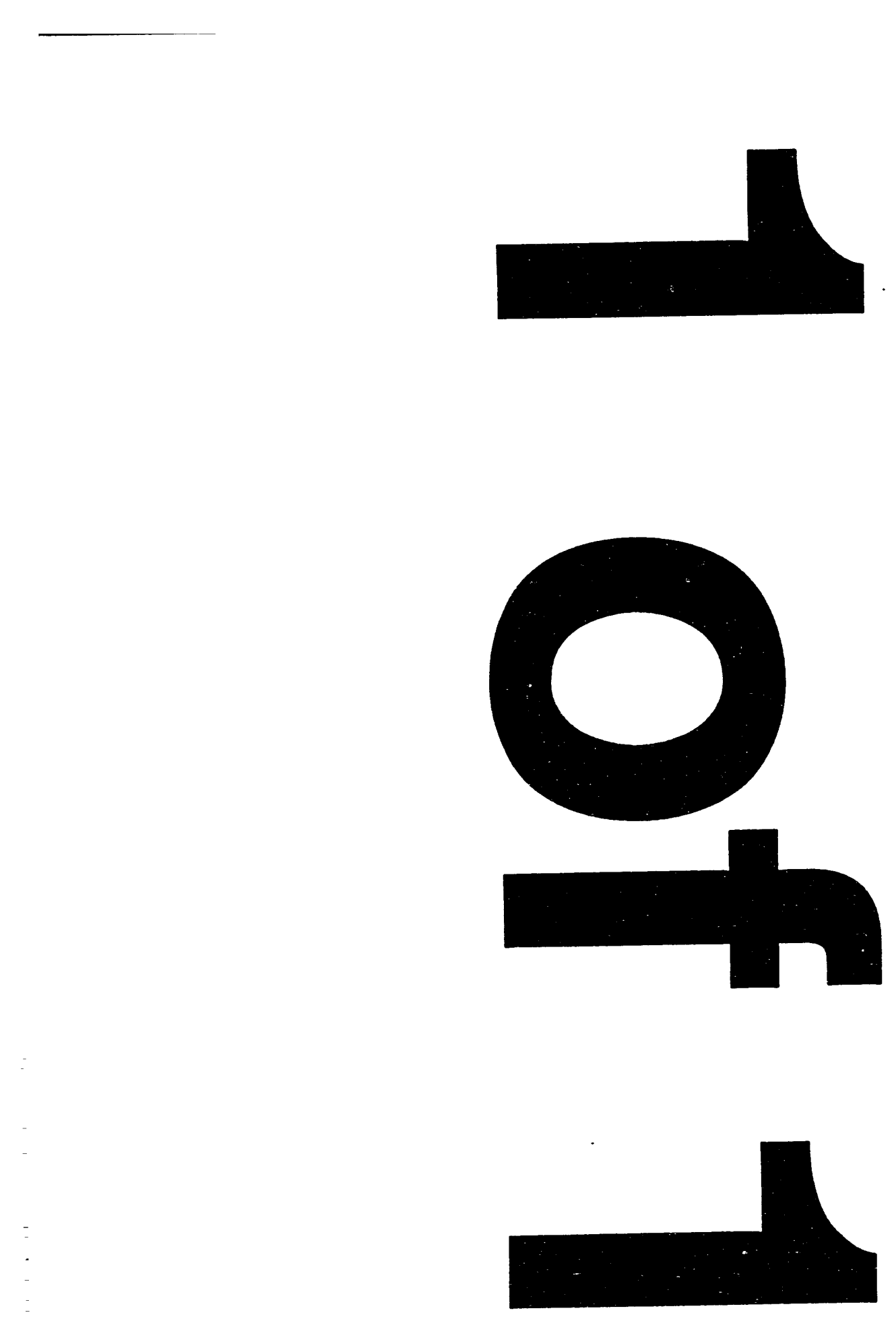
LA-UR- $94-418$

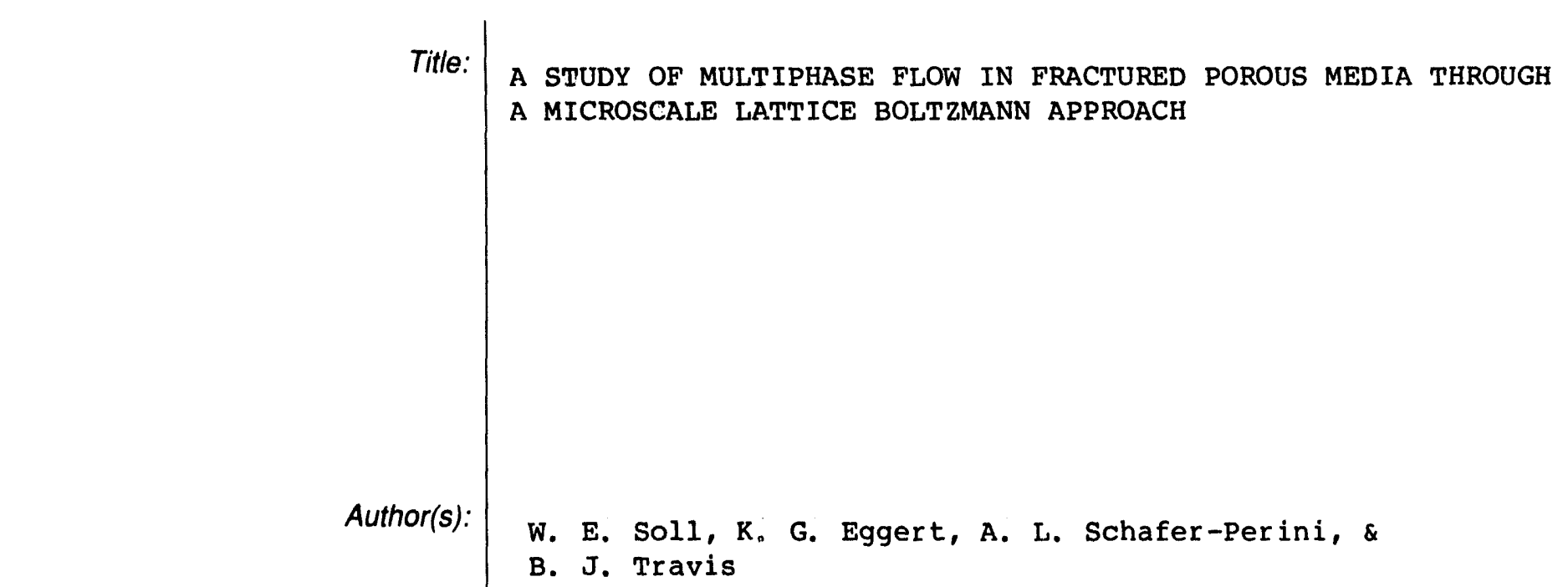

Submitted to: Computational Methods in Water Resources Heidelberg, Germany

July 19-22, 1994

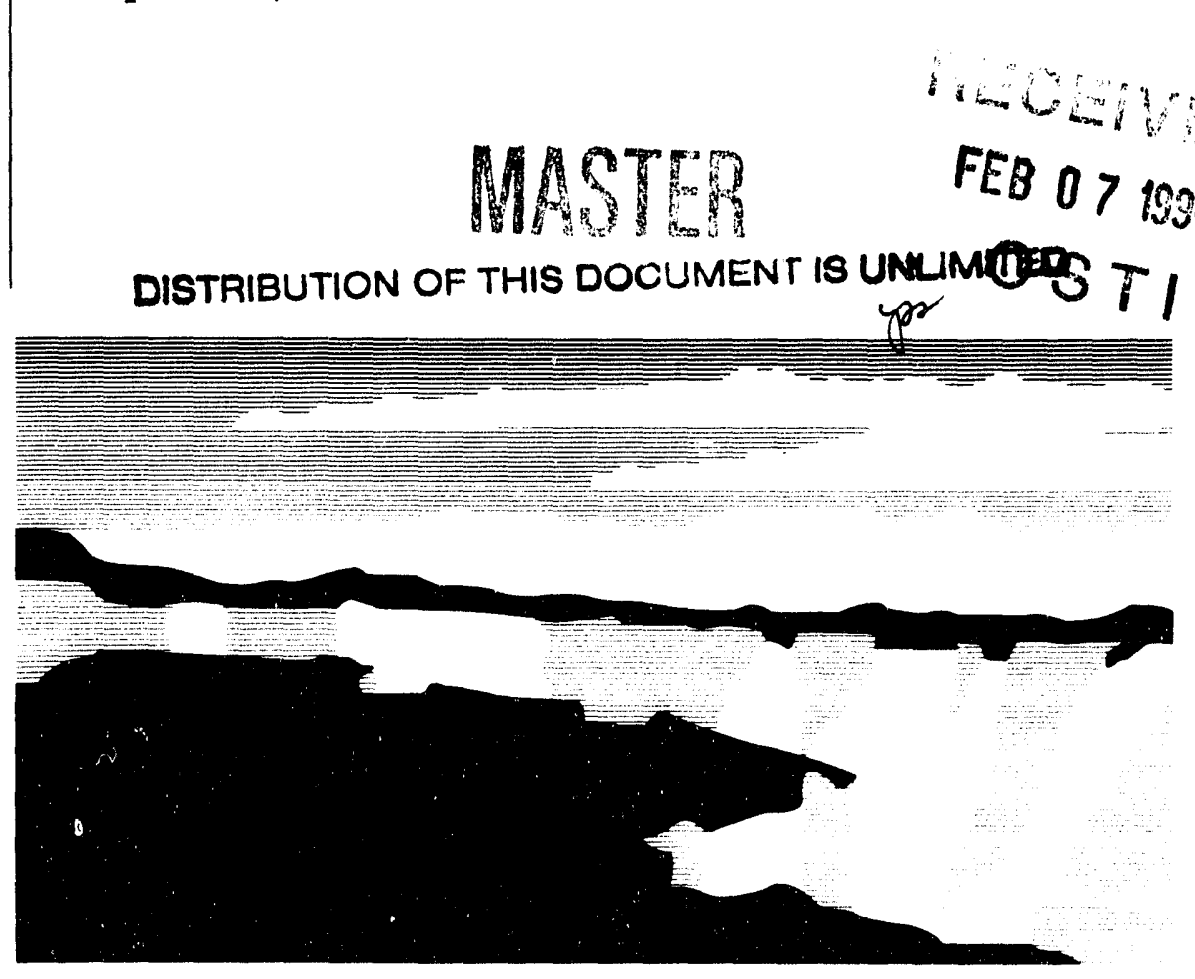

Los Alamos National Laboratory, an affirmative action/equal opportunity emplóyer, is operated by the University of California tor the U.S. Department of Energy under contract W-7405-ENG-36. By acceptance of this article, the publisher recognizes that the U.S. Government retains a nonexclusive, royalty-free license to publish or reproduce the published form of this contribution, or to allow others to do so, for U.S. Government purposes. The Los Alamos National Laboratory requests that the publisher identify this article as work performed under the auspices of the U.S. Department of Energy 


\title{
A STUDY OF MULTIPHASE FLOW IN FRACTURED POROUS MEDIA USING A MICROSCALE LATTICE BOLTZMANN APPROACH
}

\author{
W.E. Soll ${ }^{\ddagger}$, K.E. Eggert ${ }^{\ddagger}$, D.W. Grunau $\ddagger$, and A. L. Schafer-Perini ${ }^{\dagger}$ \\ ‡Earth and Environmental Sciences ${ }^{+}$ \\ Los Alamos National Laboratory \\ Los Alamos, NM, 87545 \\ USA
}

$\dagger$ E. G. \& G. Idaho, Inc.

2251 North Blvd.

Idaho Falls, ID, 83401

USA

The lattice Boltzmann technique has been shown to be an efficient and reliable approach to modeling single- and multi- fluid flow in porous media systems. The flexibility of this approach in discretizing the pore/solid space means it is particularly well suited to capturing fluid behavior, fluid-fluid interactions, and fluid-solid interactions at the scale of the individual pores. Such flexibility readily lends itself to studying processes occurring at physical interfaces, such as between a fracture and the surrounding porous matrix. Here we present pore-level simulations of fluid flow through a fracture embedded in an unsaturated matrix. Simulations are run on the massively parallel Connection Machine 5 (CM-5) using the two-fluid, two-dimensional lattice Boltzmann flow simulator developed at Los Alamos National Laboratory. We look at the effect of pressure gradients and initial matrix saturation on infiltration into the matrix and fluid flow along the fracture.

\section{INTRODUCTION}

The hydrogeologic environment at Los Alamos presents a challenge to current groundwater modeling capabilities. The topography consists of mesa tops and canyon bottoms. Beneath the mesas lies a thick $(\sim 260 \mathrm{~m})$ vadose zone made up of layers of volcanic tuff. This tuff is moderately permeable, $0.1-1$ darcy, has a very low moisture content, typically about $5 \%$, and very high capillary retention. Fractures, mostly vertical, are present in many of the tuff layers. The climate is semi-arid, with precipitation concentrated in the summer months, often in the form of short duration, high intensity events. Concerns about the relationship between precipitation on these mesa tops (some of which contain waste sites) and the deep aquifer have called attention to the limitations of our understanding of such systems. Does water enter the fracture and travel rapidly to the saturated zone? Or is water in the fracture imbibed into the matrix so fast that fractures do not provide fast flow paths? Similar concerns regarding groundwater flow in unsaturated, fractured media exist at other sites as 
well (e.g., Yucca Mountain, NV; Idaho National Engineering Laboratory, ID).

To model this problem computationally it is necessary to have a host of information about $t^{\prime}$ le system. We must have information about the porous matrix: its porosity, permeability, relative permeability, and saturation. We must have information about the fractures: location, aperture, extent, permeability, saturation, surface condition. Finally, we need to know something about how the fracture and matrix communicate, and how the water actually moves down the fracture. The difficulty is that much of this information is not available. In particular, our understanding of how the fracture and matrix interact is still quite limited. Similar concerns apply to saturated systems, in modeling diffusion processes between fracture and matrix.

There are a few different approaches currently being applied to modeling flow and transport in fractures. The most common approach models the system as a single, effective porous medium. The system is modeled as a homogeneous porous medium with constitutive characteristics that are some average of the fracture and matrix values. This approach has been shown to be fairly accurate for saturated systems with frequent, regular, and closely space fractures. However, this approach does not explicitly address fracture-matrix interaction. Another approach treats the system as a network of fractures. In this approach it is commonly assumed that all flow occurs in the fractures, and that the matrix is effectively impermeable. Some models assume that the fractures have perfectly parallel sides, and are uniformly spaced through the matrix. If the water is then assumed to be transported as a slug, semi-analytic solutions are available for predicting how far it will travel down the fracture, and how far it will penetrate into the matrix. If we choose not to make so many assumptions, we might use a numerical model that simulates the system as a dual porosity (DP) or dual porosity/dual permeability (DPDP) medium. In DP models the matrix acts solely as storage, with all flow occurring in the fractures The DPDP model allows for flow in both the matrix and the fractures. The major deficiency with the existing DP and DPDP models are that, currently, the fractures are characterized as a high permeability porous medium because of the lack of detail about how fluids are transported in fractures. This results in a model that is actually only a layered porous media system.

Regardless of which of the computational approaches are chosen from the above selection, many simplifying assumptions are made that allow the user to pretend the system is very much like a simple porous medium. Details about the fracture-matrix interface are lost. Weathering of fracture surface, or flow only along fracture walls only are ignored. Are these models truly capturing the nature of flow and transport in fractured systems? It is hard to know because of the lack of supporting experimental information. However, there is an alternative approach to obtaining answers to the many questions that have been posed in these first few paragraphs. The alternative is to study the systems at the micro-scale, through existing numerical techniques that apply fundamental principles of flow. Through these approaches, we can "observe" how the fluids distribute themselves, and apply what is learned toward improving the characterization of the physical domain and fluid-pore space interactions in the continuum scale models. 
The lattice gas (LG) / lattice Boltzmann (LB) technique has been shown to reproduce correct hydrodynamics in complex porous media through modeling of "first principles" of physics. The LB/LG method discretizes the pore space at the microscopic level, and uses simple, local, parallel operations to move particles through the lattice such that the macroscopic, averaged properties of the system reproduce the physical phenomena that satisfy the partial differential equations of interest. In the case of subsurface flow the PDEs of interest are the Navier-Stokes equations. The natural parallelism of the computational process allows rapid and accurate computation of flow fields in arbitrarily complex pore spaces utilizing massively parallel computing technology.

The ability of the LB/LG methods to handle arbitrary boundaries and multiple fluids makes them natural candidates for studying the fracture-porous matrix system described in the introductory paragraphs. Because of the nature of the LB method, it is not necessary to make any modifications to handle cases of fracture flow and fracture-matrix interactions. The proper flow physics are naturally handled in both the large volume fractures and the tortuous porous matrix, and no arbitrary boundary is imposed between the fracture and matrix.

\section{History of lattice gas / lattice Boltzmann methods}

The basic lattice gas automata model (Frisch, Hasslacher, and Pomeau, 1986; Frisch et al., 1987) consists of numerous identical particles moving on a regular lattice (a triangular lattice in 2-D and a face-centered hypercubic (FCHC) lattice in 3-D). Each particle has the same mass, momentum, and kinetic energy. Particles may occupy only the links of the lattice, and have a unit velocity corresponding to the direction of the occupied lattice link. An exclusion rule, used to minimize storage requirements, states that only one particle may occupy any given link. These rules result in a binary system, in which each lattice link is occupied (1) or empty (0). At each time step there are two stages in the process of updating the system state: advection and collision. In the advection process, a particle moves from its present site to the nearest neighbor site in the direction of the velocity vector. In the collision process, particles at each site interact, and particle momentums are redistributed at the same site such that particle number and total momentum are locally conserved. The lattice Boltzmann approach is an extension of the LG approach that uses real numbers to represent particle distributions instead of binary values.

In either LG or LB continuum scale flow fields (i.e. velocity or pressure) are obtained through averaging over a number of the lattice sites. The LB approach eliminates the need for spatial and temporal averaging that is necessary for obtaining accurate velocity and pressure fields in the LG model. Another important advantage of the continuum description of the LB method as compared to the Boolean LG models is that in the LB approach much of the statistical noise that arises in the system is eliminated.

The basic LB approach will not be described in any more detail here, as it would take more space than we are allowed, and is thoroughly described in a number of excellent references. The reader is directed to the original LG derivations referenced above, as well as to Grunau (1993) for a good discussion of both LG and LB method derivations. McNamara and 
Zanetti (1988) is the original publication on development of the LB approach from lattice gas methods. Gunstensen et al. (1991) and Grunau et al. (1993) offer excellent descriptions of LB models for two-fluid systems. Soll et al. (1994, in this proceedings) present a general discussion of the latest capabilities of the LANL LB model used for this work, and cover some of the other areas to which this model is being applied.

\section{FRACTURE FLOW SIMULATIONS}

In this section, simulations are presented from a number of different simple fracture configurations. The generic system is meant to approximate infiltration of water into a fracture and subsequent downward movement through both the fracture and the porous matrix, where the medium is unsaturated. Fluid enters into the fracture from the left side of the domain, but is restricted from entering the matrix on the inflow face. Outflow on the right side of the domain is open across the entire domain height. The flow is driven by a uniform pressure difference across the system. The entering fluid is strongly wetting, and has a higher viscosity than the resident fluid, which, by comparison, is non-wetting. Gravity is neglected in these simulations, although the LB code is capable of accommodating gravitational forces. In all of the figures the solid is shown in black, the wetting fluid (WF) is shown in white, and the non-wetting fluid (NWF) is shown in gray. The infiltrating fluid in all of the following simulations is the wetting fluid and always advances from left to right.

The simplest fracture is a pair of parallel, impermeable plates. This model is Poiseuille flow and, though not very realistic, provides an easy means of verifying proper model response. Figure 1 shows a step from a simulation of two-fluid flow between parallel plates. We observe the expected curved front. Figure 2 shows the corresponding velocity profile. When two immiscible fluids flow simultaneously, side by side, the velocity profile changes to reflect the difference in viscosities. The dotted line shows the actual velocity profile across the channel width overlaid on each of the individual velocity distributions.

Similar simulations have been run for "fractures" with parallel, impermeable walls into which a wall roughness has been introduced. Figure 4 shows the fluid distribution in this rough-walled system. Note that the front retains the curved profile and much of the symmetry at the leading edge, where the flow front is far enough from the wall not to be influenced by the roughness. However, the flow is somewhat retarded with respect to the smooth walled system (Fig. 1), and residual NWF occupies some of the roughness volume behind the advancing WF front.

The next level of complexity of pore space is having a porous matrix bounding the parallel fracture walls. In this configuration the porous matrix is from a digitization of a micromodel. A $512 \times 512$ subsection is taken from the micromodel space, and a parallel "fracture" is introduced down the middle of the domain. The fracture width is 32 lattice sites, sufficiently wide to balance the relatively large pore widths in the micromodel and produce a fracture with greater permeability than that of the pore space. For the initial analysis of fracture-matrix interactions we chose to run the simulations in a 2-D pore space to reduce the computational burden and allow easy visualization. 3-D systems will be 


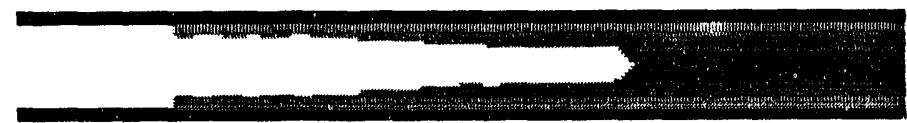

Fig. 1. Wetting fluid (white) displacing non-wetting fluid (gray) between a parallel plate "fracture."

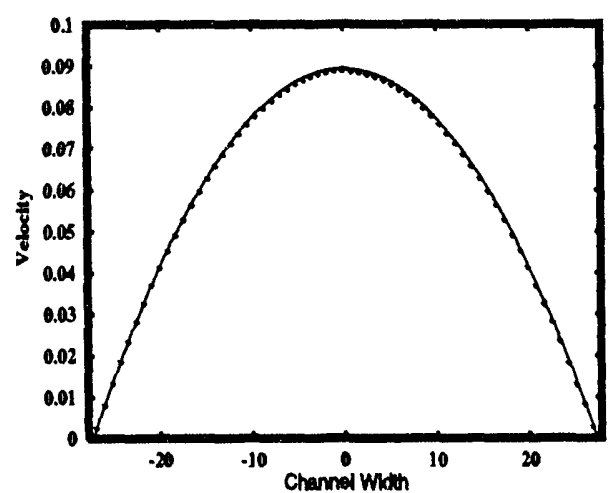

Fig. 2. Velocity profile for a single fluid flowing between parallel plates.

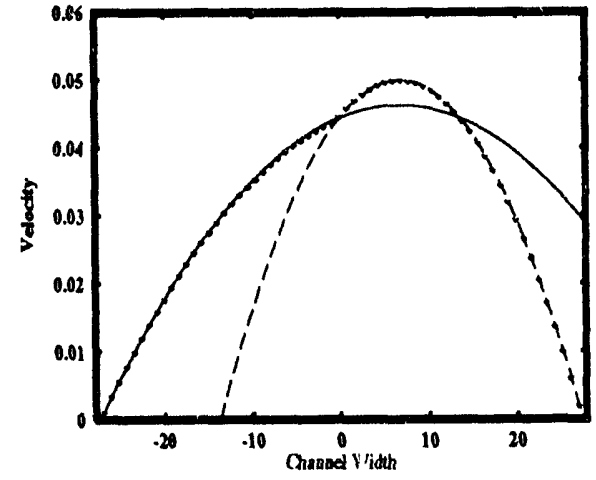

Fig. 3. Velocity profile for flow between parallel plates with two concurrently flowing fluids.

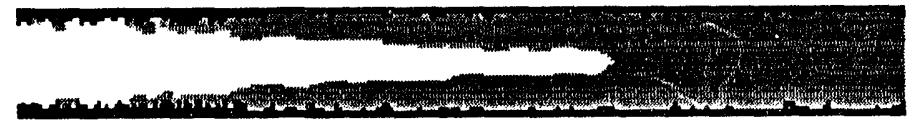

Fig. 4. Wetting fluid (white) displacing non-wetting fluid (gray) between a rough walled parallel "fracture". 
addressed in future work.

Simulations were run to compare infiltration into the matrix from the fracture for different forcing pressures across the fracture length. Figure 5 shows a comparison of two systems in which the pressure gradient in Figure 5a is twice that in Figure 5b. Both figures represent the fluid distributions after the same number of time steps. The WF has infiltrated significantly farther into the matrix in Figure 5a as in Figure 5b. A similar WF saturation as in Figure 5a for the higher forcing pressure is obtained for the lower forcing pressure after slightly more than twice the number of time steps, suggesting that the matrix infiltration is largely being governed by the flow rate.

The model was also used to analyze the effect of different initial fluid saturations in the matrix on fluid movement between fracture and matrix. Initial conditions with non-zero WF saturation are generated by assigning a saturation fraction, which the code distributes homogeneously through the pore space. The system is then allowed to equilibrate under no external forces. During equilibration the fluids separate due to interfacial forces, and the wetting fluid migrates to the solid surface. The equilibrium state then provides the initial fluid distribution for the flow simulations.

Figure 6 shows a comparison of infiltration at two different initial matric wetting-fluid saturations. The system in Figure 6a started with an initial WF saturation of zero, while the system in Figure $6 \mathrm{~b}$ had an initial matric WF saturation of approximately 0.40 . The displacement in these simulations is viscous-dominated as a result of choosing a relatively large driving pressure gradient. In this comparison the WF in the initially NWF saturated system (Fig. 6a) has penetrated farther into the matrix near the fracture entrance than that in the partially WF saturated system (Fig. 6b). However, the WF has penetrated more rapidly down the fracture in Figure $6 \mathrm{~b}$ than in Fig 6a., and the WF has penetrated into the matrix along more of the length of the fracture. A simulation run with an initial WF saturation of 0.10 followed the same trend. The difference in the WF penetration into the matrix is partly a result of reduced capillary pressure and relative permeability in the matrix at the higher WF saturation. A second factor in the reduced matrix penetration in Figure $6 \mathrm{~b}$ is the change in effective pressure drop across the fracture in the partially saturated system as the WF flow volume in the fracture increases.

Note that Figure 6a and Figure 5a are from the same simulation at 5000 and 10000 time steps, respectively. Comparing these two figures shows that, once the WF crosses the entire fracture, the distance along the fracture that is saturated by WF increases slowly, while the distance the WF has penetrated the matrix near the entrance grows measurably. The increase in WF saturation in the matrix is small relative to the WF flux through the fracture.

\section{THE NEXT STEP}

We have presented preliminary results of a study to characterize flow through a porous system composed of fractures and porous matrix. The data presented here were limited to some of the simplest pore space configurations. The next step is to move to more realistic 

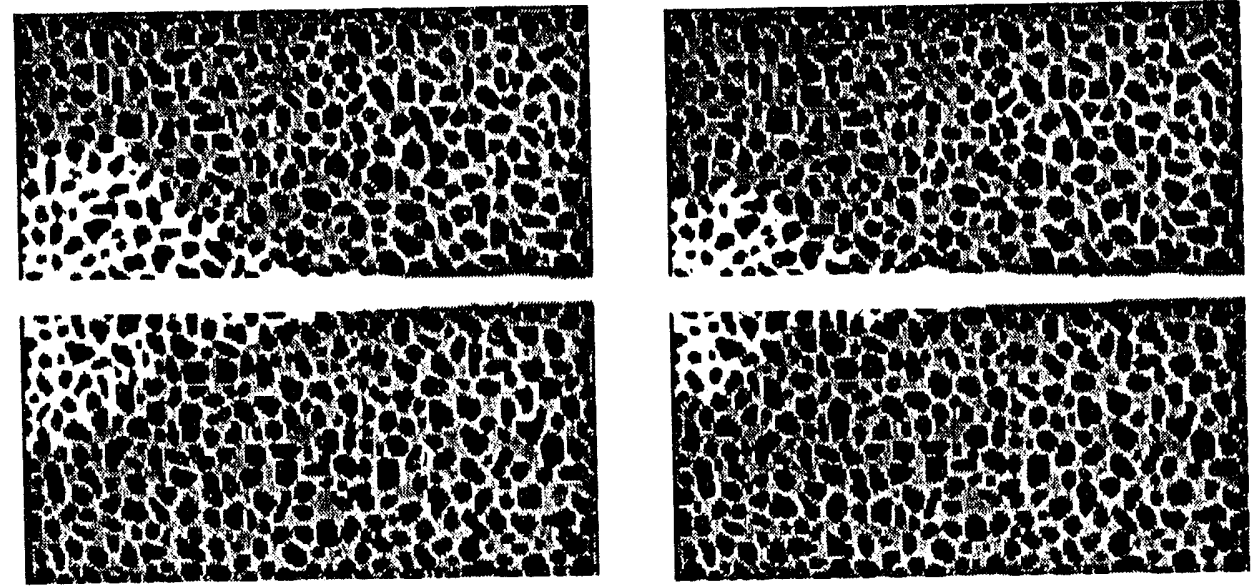

Fig. 5. Effect of pressure gradient on fracture-matrix llow. White is wetling fluid. Gray is non-wetting lluid. Gradient in (a) is twice as large as $(h)$.
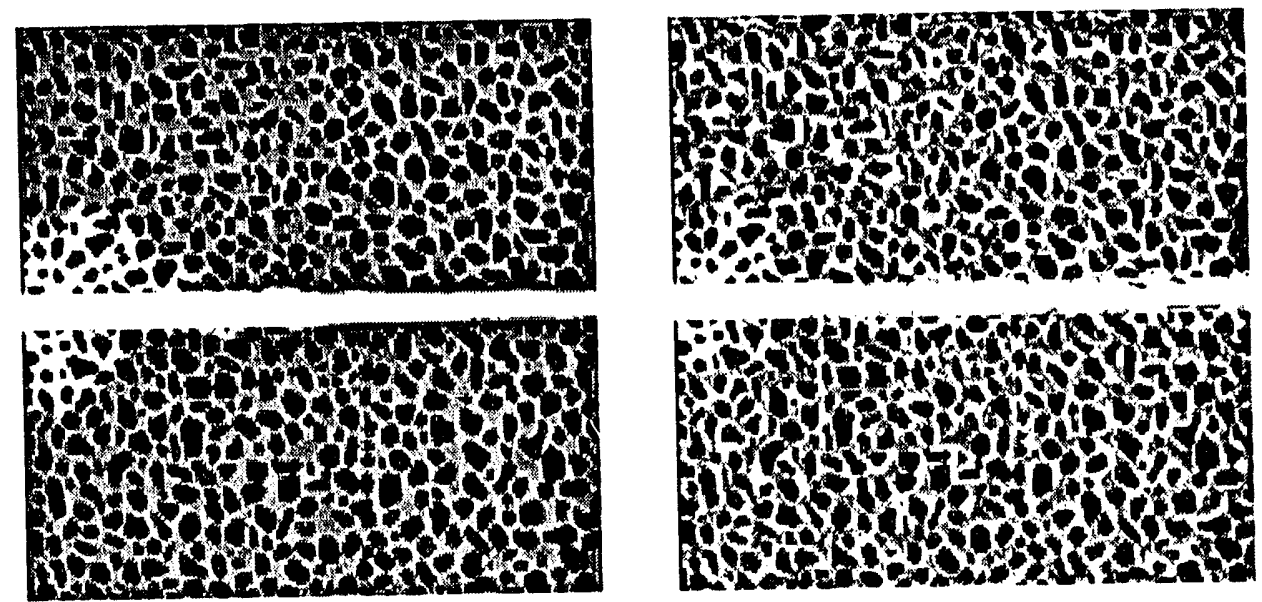

Fig. 6. Effect of initial wetting fluid matrix saturation on fracture-matrix llow. Initial matrix saturation of wetting fluid (a) is $(0 .(),(b)$ is $(0.5)$. 
physical systems. To do this we must be able to generate such systems. We intend to apply a pore space generation code, that has been developed by the authors, to generate 3-D pore spaces with more complex fractures. We also hope to obtain high resolution digital images of a fractured porous medium. Systems with both single and multiple fractures will be considered. Simulation results will be compared against available analytic or semi-analytic solutions, as well as any available experimental data.

\section{ACKNOWLEDGEMENTS}

This work has been supported by the Los Alamos National Laboratory which is operated by the University of California for the U.S. Department of Energy. The authors wish to acknowledge the Advanced Computing Laboratory of Los Alamos National Laboratory, Los Alamos, NM 87545. This work was performed on computing resources located at this facility. The authors also thank S.Y. Chen for code concept development.

\section{REFERENCES}

Chen, S.Y., G.D. Doolen, K. Eggert, D. Grunau, and E.Y. Loh. (1991). "Lattice Gas Simulations of One and Two-Phase Fluid Flows Using the Connection Machine-2," in A.S. Alves (ed.), Discrete Models of Fluid Dynamics, World Scientific. pp. 232-249.

Frisch, U., B. Hasslacher, and Y. Pomeau (1986). "Lattice-Gas Automata for the Navier-Stokes Equation". Physical Review Letters, 56, 1505-1508.

Frisch, U., D. d'Humieres, B. Hasslacher, P. Lallemand, Y. Pomeau, and JP. Rivet. (1987). "Lattice Gas Hydrodynamics in Two and Threc Dimensions". Complex Syst, 1. pp. 649-707.

Grunau, D.W. (1993). Lattice Methods for Modeling Hydrodynamics. PhD Dissertation, Colorado State University, Dept. of Math.

Grunau, DW., SY. Chen, and K. Eggert (1993). "A Lattice Boltzmann Model for Multi-phase Fluid Flows". Phys. Fluids A.,5(10). pp. 2557-2562.

Gunstensen, A.K., D.H. Rothman, S. Zaleski, and G. Zanetti. (1991). "Lattice Boltzmann Model of Immiscible Fluids". Phys. Rev. A., 43(8). pp. 4320-4327.

McNamara, G.R., and G. Zanetti (1988). "Use of the Boltzmann Equation to Simulate Lattice-Gas Automata”. Phys. Rev. Lett., 61(20). pp. 2332-2335.

\section{DISCLAIMER}

\footnotetext{
This report was prepared as an account of work sponsored by an agency of the United States Government. Neither the United States Government nor any agency thereof, nor any of their employees, makes any warranty, express or implied, or assumes any legal liability or responsibility for the accuracy, completeness, or usefulness of any information, apparatus, product, or process disclosed, or represents that its use would not infringe privately owned rights. Reference herein to any specific commercial product, process, or service by trade name, trademark, manufacturer, or otherwise does not necessarily constitute or imply its endorsement, recommanthe views mendation, or favoring by the United States Government or ans state or reflect those of the United States Government or any agency thereof.
} 

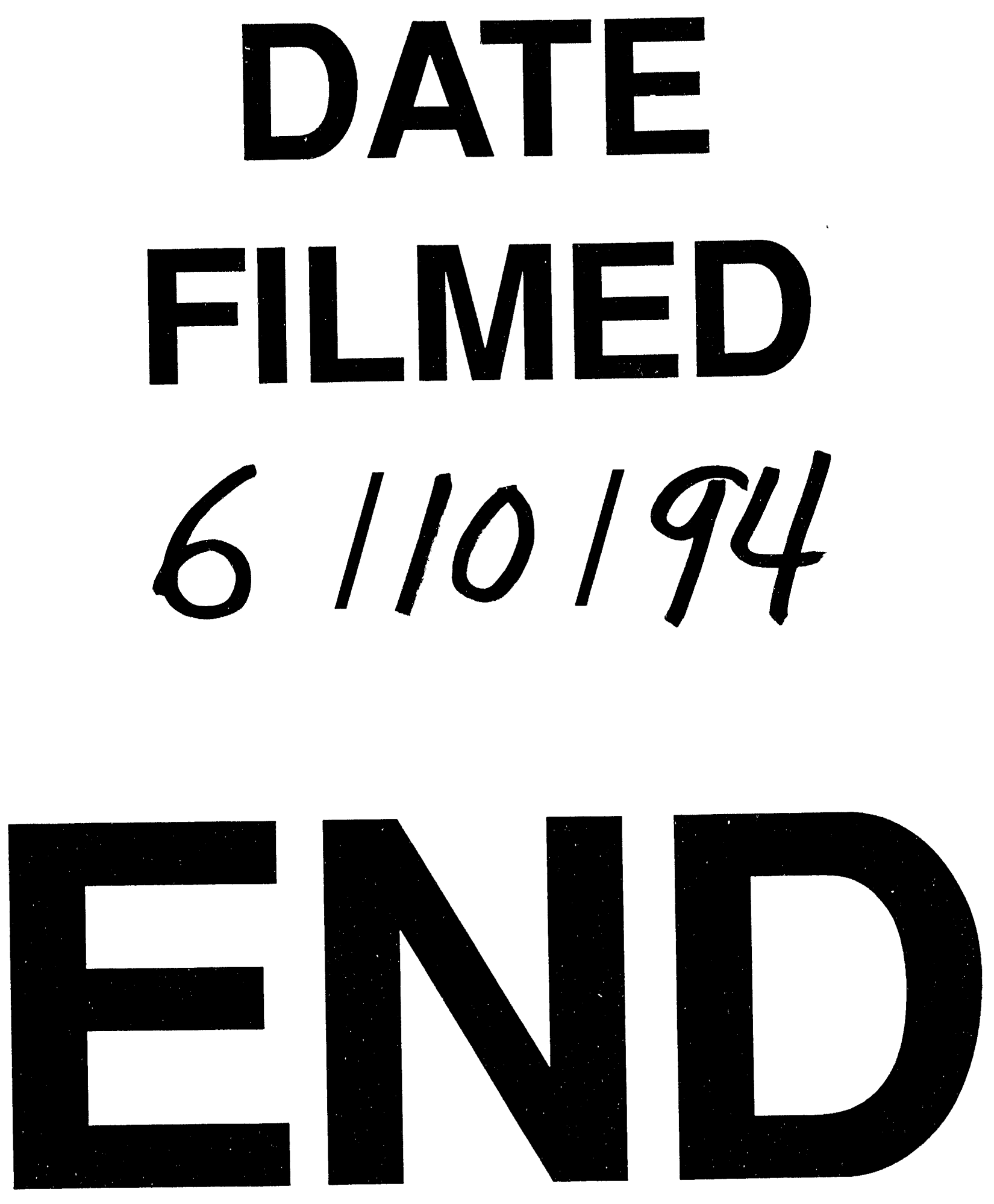
+2.... 